Research Article

\title{
Cavitation Detection in Centrifugal Pump Based on Interior Flow-Borne Noise Using WPD-PCA-RBF
}

\author{
Liang Dong $\mathbb{D}^{1,2}$ Kan Wu, ${ }^{1}$ Jian-cheng Zhu, ${ }^{1}$ Cui Dai $\left(\mathbb{D},{ }^{3}\right.$ Li-xin Zhang, ${ }^{1}$ and Jin-nan Guo ${ }^{1}$ \\ ${ }^{1}$ Research Center of Fluid Machinery Engineering and Technology, Jiangsu University, Zhenjiang 212013, China \\ ${ }^{2}$ Sichuan Provincial Key Lab of Process Equipment and Control, Sichuan University of Science \& Engineering, \\ Zigong 643000, China \\ ${ }^{3}$ School of Energy and Power Engineering, Jiangsu University, Zhenjiang 212013, China \\ Correspondence should be addressed to Cui Dai; daicui@ujs.edu.cn
}

Received 18 March 2019; Accepted 21 April 2019; Published 12 May 2019

Academic Editor: Adam Glowacz

Copyright (C) 2019 Liang Dong et al. This is an open access article distributed under the Creative Commons Attribution License, which permits unrestricted use, distribution, and reproduction in any medium, provided the original work is properly cited.

\begin{abstract}
Cavitation detection is particularly essential for operating efficiency and stability of pumps. In this work, to improve the accuracy and efficiency of identification, an approach combining wavelet packet decomposition (WPD) with principal component analysis (PCA) and radial basic function (RBF) neural network is introduced to detect the cavitation status for centrifugal pumps. The cavitation performance and interior flow-borne noise are measured under three different flow conditions. Then, time-frequency domain analysis is performed on the interior flow-borne noise signal using WPD, and the energy coefficient of each node is calculated to determine the optimal decomposition frequency band. Six-feature parameters are extracted based on frequencydivision statistics, including three time-domain features and three wavelet packet features. After that, the PCA is applied for dimensionality reduction. Finally, three cavitation statuses of noncavitation, inception cavitation, and serious cavitation are identified adopting RBF neural network. The results show that the comprehensive identification rate of the proposed method for three cavitation statuses reaches $98.2 \%$ with low identification error. The method based on interior flow-borne noise analysis can be well applied for on-line monitoring and diagnosis of pump industry.
\end{abstract}

\section{Introduction}

As the most widely used general machinery, pump plays a vital role in the fields of hydraulic transportation, water irrigation, flood control and drainage, and hydropower generation. However, for centuries, the cavitation within a pump has been restricting the development of the pump industry, which not only limits the efficient operating range of the pump but also affects the operational reliability of the pump system. The cavitation can appear within the entire range of operating conditions in pumps. Therefore, it is necessary to detect the cavitation status to avoid spreading to other parts of the pump system.

The signal-based approaches, such as vibration, acoustic emission, electrical currents, and interior flow-borne noise, are common approaches in cavitation detection in centrifugal pumps [1]. Wang and Chen [2] presented a method for a centrifugal pump based on vibration signals. In the method, a wavelet transform was used to extract features from measured vibration signals, and a linearized neural network was utilized to distinguish cavitation types. Sakthivel et al. [3] carried out condition monitoring of a monoblock centrifugal pump using vibration signals. The statistical features were transformed using dimensionality reduction techniques and then the reduced set was applied to a decision tree to classify different modes of the pump. Alfayez et al. [4] presented a case study where acoustic emission has been applied for detecting incipient cavitation and determining the best efficiency point (BEP) of a $60 \mathrm{~kW}$ centrifugal pump. Farokhzad and Ahmadi [5] concentrated on a procedure for prediction of cavitation using acoustic signals and multilayer perceptron neural network. Durocher and Feldmeier [6] used stator currents to detect cavitation status in a centrifugal pump. Hernandez-Solis and Carlsson 
[7] measured motor current and voltage signals for different operating points of the pump and studied the correlation between the cavitation phenomena and the power of the motor. The main challenge of using vibration, acoustic emission, and electrical current signals is that the measured signals are so complex to identify the relationship between characteristic parameters and cavitation status due to the complexity of the pump.

In fact, the interior flow-borne noise induced by cavitation in the pump is mainly a monopole noise source with strong radiation efficiency and impact characteristic. And the centrifugal pump presents particular cavitation noise characteristics in normal operating condition compared with that upon inception cavitation. Chudina [8] utilized the interior cavitation noise spectra to detect the onset of cavitation in a centrifugal pump. The results show that there is a discrete frequency component, which is strongly dependent on the cavitation process and its development, and the noise spectra can also be used to determine the NPSH required or the critical value, representing the upper limit of permissible pump operation without cavitation. In order to study the change rules of interior flow-borne noise with the development of cavitation, Dong et al. [9] optimized the calculation accuracy using computational fluid dynamics combined with the Lighthill acoustic analogy. The results show that, with the development of cavitation, the sound pressure level (SPL) at axial passing frequency (APF) and the frequency bands of $10 \mathrm{~Hz} \sim 100 \mathrm{~Hz}$ and $1000 \mathrm{~Hz} \sim 3000 \mathrm{~Hz}$ show an increasing trend, while it decreases at blade passing frequency (BPF) and its harmonics. At the initial cavitation, the frequency band between $1000 \mathrm{~Hz}$ and $3000 \mathrm{~Hz}$ shows the highest sensitivity for cavitation status detection.

However, the cavitation noise in pumps is a highfrequency continuous spectrum signal, and the frequency spectrum analysis based on Fourier transform has insurmountable limitations for cavitation detection with strong shock catastrophe and nonstationary characteristics [10]. Wang et al. [11] conducted the cavitation noise evaluation using wavelet packet decomposition (WPD) and described the singular characteristics of cavitation noises using the extreme amplitude values in the transformed domain. Two new parameters were suggested to describe the characteristics of cavitation noises under different cavitation degrees. Application to real data analysis shows that the presented method provides more information on abrupt changes in cavitation noises signals, and is useful in identification of cavitation development stage. Wang and Chen [12] proposed a sequential diagnosis method using fuzzy neural network method to distinguish at an early stage on the basis of the possibilities of symptom parameters.

Moreover, it is still one difficult problem that how to detect cavitation status in pumps more efficiently. The eigenvalues, such as the average, root mean square, and standard deviation, were widely used in the feature extraction. However, the cavitation characteristics are distributed over a wide frequency range and the characteristics of different frequency bands varies. The use of single eigenvalue in a full-frequency band cannot reflect the change rule of the signal at different frequency bands before and after cavitation. So, it is difficult to determine the eigenvalues and their thresholds that can accurately identify the cavitation states [13].

In this paper, to overcome these difficulties, a multiresolution cavitation status detection method for a centrifugal pump is proposed based on the interior cavitation noise signal. First, the wavelet packet decomposition (WPD) is employed to extract the decomposition frequency band characteristics. The principal component analysis (PCA) is applied to reduce dimensionality and remove redundancy. Then, three cavitation statuses including noncavitation, inception cavitation, and serious cavitation are identified using radial basic function (RBF) neural network. Finally, the classification and recognition effect of the proposed method for the three cavitation statuses in centrifugal pumps are analyzed.

\section{Theory of WPD-PCA-RBF}

To improve the recognition accuracy and efficiency of cavitation status in pumps, a cavitation detection method based on wavelet packet decomposition (WPD), principal component analysis (PCA), and radial basic function (RBF) neural network is proposed. The structure of the proposed method is presented in Figure 1 by means of a flow diagram, which describes the different stages of the methodology.

The algorithmic details for each major step in the method are as follows:

(1) Detect different types of cavitation status by cavitation performance experiment.

(2) Collect original liquid-borne noise data under different cavitation status.

(3) Solve the energy coefficient on each node and determine the research nodes with larger energy coefficients by WPD.

(4) Extract the eigenvalue of each research node and compose the eigenvalue matrix. Normalize the eigenvalue matrix due to large magnitude difference between eigenvalue elements.

(5) To improve the recognition speed, PCA is employed to obtain the principal component matrix with dimensionality reduction.

(6) Input the principal component matrix of training sample into RBF as an input layer, determine the number of hidden layer nodes and RBF structure, and then obtain the cavitation status recognition result on the output layer. Calculate the error between the desired output and actual output until the training samples are fully trained and meet the network accuracy requirement or the iteration number is reached. Eventually, establish an ideal RBF network.

(7) Input the principal component matrix of testing sample into the ideal RBF network and output the diagnosis result.

2.1. WPD. WPD is a mathematical method that can provide a multiscale time-frequency analysis by transforming signals 


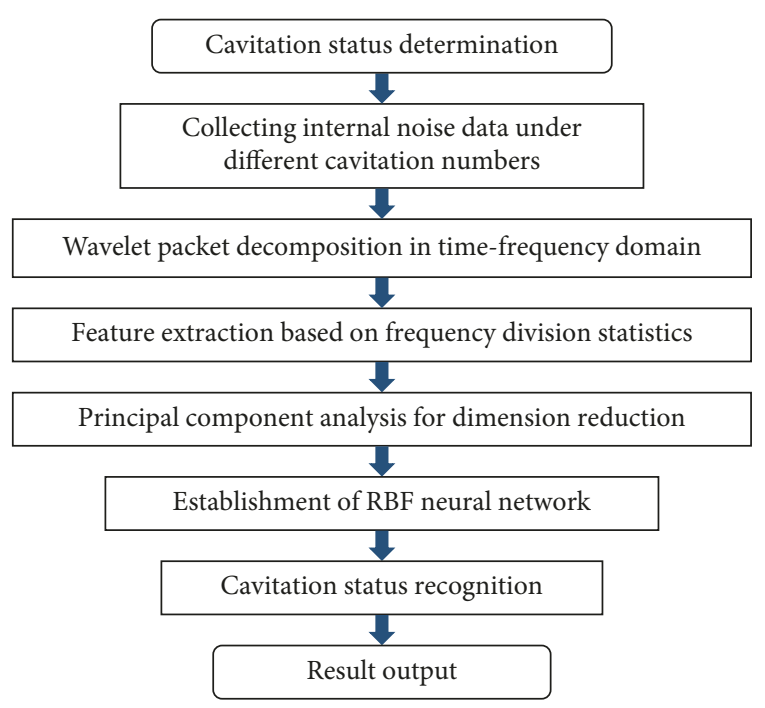

FIgURE 1: Flow chart of the cavitation detection method.

from time domain into equal-width sub-band frequency domain. With orthogonal transform, the WPD has the added benefit that the energy in the signals can be preserved and partitioned among the coefficients.

Given the sampling frequency of the signal, $\{f(t)\}$ becomes $f(s)$. Based on the sampling theorem, the maximum analysis frequency of the signal is $f(s) / 2$. After the $j$-layer wavelet packet transform applied on the signal, the frequency band $\{0-(f(s) / 2)\}$ is divided into $\left\{2^{j}\right\}$ equal-width segments, that is, the width of each sub-band is $f(s) /\left(2 \times 2^{j}\right)$. Correspondingly, the coefficient at each layer $C_{i, k, n}$ is obtained, where $i=2,3, \ldots, j, k=0,1,2, \ldots, 2^{i}-1$ and $n$ is the location index. According to Parseval energy equation, the signal energy can be expressed as the integral of amplitude square over the entire time domain. The coefficient is used to represent the similarity between the wavelet packet function and the real signal. Therefore, the coefficient $C_{i, k, n}$ can be used to represent the signal energy as follows [14]:

$$
f(t)^{2}=\sum_{k=0}^{2^{j}-1}\left|C_{j, k}\right|^{2}
$$

where $j$ and $k$ are the number of decomposition layer and frequency band, respectively.

In the present study, after appropriate determination of wavelet basis function and decomposition scale $j$, a $j$-layer wavelet packet transform was then applied on the signal. Because the orders of magnitude of the eigenvalue elements differ greatly, normalization is performed to make sure that the input data are within an appropriate range. The wavelet packet energy of the signal on all frequency bands is calculated by formula (1), and the wavelet packet energy on each frequency band is normalized, that is

$$
\begin{aligned}
& T_{j, k}=\sum_{n=1}\left|C_{j, k, n}\right|^{2}, \\
& T_{j, k}^{\prime}=\frac{T_{j, k}}{\sum_{k=1}^{2 j-1} T_{j, k}},
\end{aligned}
$$

where $T_{j, k}$ is the wavelet packet energy value on the $k_{\mathrm{th}}$ frequency band and $T_{j, k}^{\prime}$ is the normalized feature vector.

2.2. PCA. PCA is a statistical procedure that uses an orthogonal transformation to convert a set of observations of possibly correlated variables into a set of values of linearly uncorrelated variables called principal components. It can be used to reduce the data dimensionality and eliminate some irregularities from the data. The reduction process is as follows [15].

Given a set of $m$ dimension feature vectors $x_{i}(i=1,2, \ldots$, $n$ ), normally $m<n$, the mean vector $\mu$ and covariance matrix $C$ are calculated by

$$
\begin{aligned}
& \mu=\frac{1}{n} \sum_{i=1}^{n} x_{i}, \\
& C=\frac{1}{n} \sum_{i=1}^{n}\left(x_{i}-\mu\right)\left(x_{i}-\mu\right)^{\mathrm{T}} .
\end{aligned}
$$

By matrix manipulation, we obtain $C y_{j}=\lambda_{j} y_{j}$, where $\lambda_{j}$ $(j=1,2, \ldots, m)$ is the eigenvalue sorted in descending order and $y_{j}(j=1,2, \ldots, m)$ is the corresponding eigenvector. The matrixes of eigenvalues and eigenvectors can be constructed as $A=\operatorname{diag}\left[\lambda_{1}, \lambda_{2}, \ldots, \lambda_{m}\right]\left(\lambda_{1}>\lambda_{2}>\ldots>\lambda_{m}\right)$ and $Y=\left[y_{1}, y_{2}\right.$, $\left.\ldots, y_{m}\right]$. To eliminate redundancy, $k(k \leq m)$ eigenvectors corresponding to $k$ largest eigenvalues are selected. A threshold $\theta$ is introduced to decide the number $k$ by computing the proportion of $k$ largest eigenvalues in all eigenvalues.

$$
\frac{\sum_{j=1}^{k} \lambda_{j}}{\sum_{j=1}^{m} \lambda_{j}} \geq \theta .
$$

Given the parameter $\theta$, the number $k$ can be determined. Then, the principal components with low dimensions are decided as $P=\left[y_{1}, y_{2}, \ldots, y_{k}\right]^{\mathrm{T}} x_{i}$.

2.3. RBF. RBF neural network is a particular type of artificial neural network (ANN) that uses radial basis functions as activation functions. In principle, it could be employed in any sort of model, and particularly suitable for solving the status recognition problems with abrupt change in signals. It consists of an input layer, a hidden layer of RBF neurons, and an output layer with one node per category or class of data $[16,17]$.

The RBF neurons store an available set of input-output pairs of vectors, called training set. Each RBF neuron will compute a measure of the similarity between the input and its training vector. The input vectors which are more similar to the training sets will return a result closer to 1 . The output of RBF neural network consists of a set of nodes, one per category that we are trying to classify. Each output node computes a sort of score for the associated category. The score is computed by taking a weighted sum of the activation values from every RBF neuron. Determining the RBF weights is called training. After training, the RBF can be 
implemented with data whose underlying statistics is similar to that of the training set.

The interpolation of a set of $N$ data points in a multidimensional space requires every one of the $D$ dimensional input vectors $\mathbf{x}^{p}=\left\{x^{p}: i=1, \ldots, D\right\}$ to be mapped onto the corresponding target output $t^{P}$. The goal is to find a function $f(\mathbf{x})$ such that $f\left(\mathbf{x}^{p}\right)=t^{p} \forall p=1, \ldots, N$. The radial basis function method introduces a set of $N$ basis functions, one for each data point, which takes the form $\phi\left\|\mathbf{x}-\mathbf{x}^{p}\right\|$, where $\phi(\cdot)$ is some nonlinear function. Thus, the $p_{\text {th }}$ function depends on the distance $\left\|\mathbf{x}-\mathbf{x}^{p}\right\|$, usually taken to be Euclidean, between $\mathbf{x}$ and $\mathbf{x}^{p}$. The output of the mapping is taken to be a linear combination of basic functions, i.e., $f(\mathbf{x})=\sum_{p=1}^{N} w_{p} \phi\left(\left\|\mathbf{x}-\mathbf{x}^{p}\right\|\right)$. The idea is to find the "weights" $w_{p}$ such that the function goes through the data points. It is easy to determine equations for the weights by combining the above equations, that is, $f\left(\mathbf{x}^{q}\right)=t^{q}$. By definition, $\mathbf{t}=\left\{t^{p}\right\}, \mathbf{w}=\left\{w^{p}\right\}$, and $\boldsymbol{\Phi}=\left\{\Phi_{p q}=\phi\left(\left\|\mathbf{x}^{q}-\mathbf{x}^{p}\right\|\right)\right\}$. The above equation can be simplified in matrix form as $\Phi \mathbf{w}=\mathbf{t}$. Then, provided the inverse of $\boldsymbol{\Phi}$ exists, we can use any standard matrix inversion technique to give $\mathbf{w}=\Phi^{-1} \mathbf{t}$. It can be shown, for a large class of basic function $\phi(\cdot)$, the matrix $\Phi$ is indeed nonsingular (and invertable) providing the data points are distinct. Once we have the weights, we have a function $f(\mathbf{x})$ that represents a continuous differentiable surface that passes exactly through each data point.

Gaussian function is one of the most commonly used basic functions [18].

$$
\phi(r)=\exp \left(-\frac{r^{2}}{2 \sigma^{2}}\right),
$$

where $r>0$ represents a distance from a data point $x$ to a centre $c$ and the variable $\sigma$ is the width parameter that controls the smoothness of the interpolating function which is always greater than zero.

\section{Experimental Investigation}

3.1. Experiment Model. The experimental model is a lowspecific speed centrifugal pump with a spiral volute in [19]. The design parameters of the centrifugal pump are design flow rate $Q_{\mathrm{d}}=12.5 \mathrm{~m}^{3} / \mathrm{h}$, design head $H_{\mathrm{d}}=74 \mathrm{~m}$, rotating speed $n=2950 \mathrm{r} / \mathrm{min}$, and specific speed $n_{\mathrm{s}}=25$. The main geometry parameters of the centrifugal pump are given in Table 1. The axial passing frequency (APF) and blade passing frequency $(\mathrm{BPF})$ of the centrifugal pump are, $49 \mathrm{~Hz}$ and $295 \mathrm{~Hz}$, respectively.

3.2. Test Rig. The experimental test rig was designed and constructed in the National Engineering and Technology Research Centre of Pump and Pumping System, Jiangsu University, as illustrated in Figure 2. The experimental system mainly consists of a centrifugal pump, a vacuum pump, a cavitation tank, a pressure stabilizer, an exhaust port, inlet and outlet pipes, inlet and outlet valves, a variablefrequency driving motor, and others. Installed at the pump outlet, there is a pressure stabilizer for stabilizing the fluid pressure in the system and preventing the temperature rise
TABLE 1: Main geometry parameters of model pump.

\begin{tabular}{lcc}
\hline & Parameter & Value \\
\hline \multirow{4}{*}{ Impeller } & Inlet diameter, $D_{1}(\mathrm{~mm})$ & 68 \\
& Outlet diameter, $D_{2}(\mathrm{~mm})$ & 228 \\
& Number of blades, $z$ & 6 \\
& Wrapping angle, $\theta / r$ & 145 \\
Volute & Outlet width, $b_{2}(\mathrm{~mm})$ & 7 \\
& Basic diameter, $D_{3}(\mathrm{~mm})$ & 245 \\
& Inlet width, $b_{3}(\mathrm{~mm})$ & 18 \\
& Discharge nozzle diameter, $d_{4}(\mathrm{~mm})$ & 32 \\
\hline
\end{tabular}

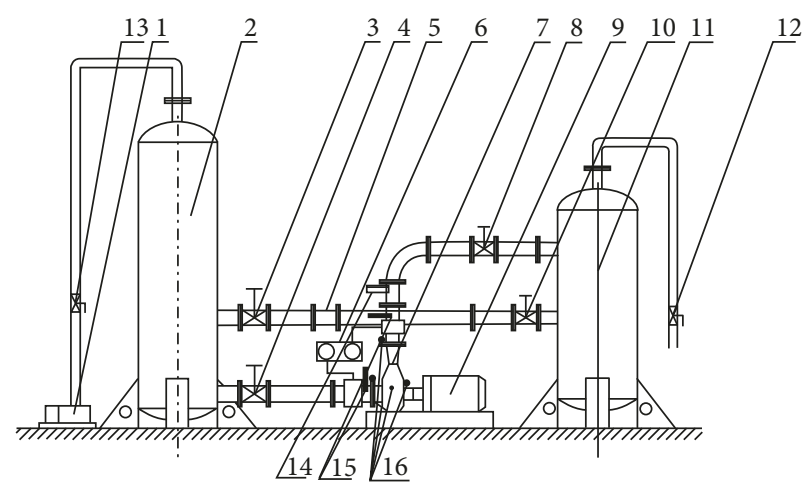

FIGURE 2: Schematic of test rig: (1) vacuum pump; (2) cavitation tank; $(3,4,8,10)$ brake valves; (5) electromagnetic flowmeter; (6) pressure transducers; (7) model pump; (9) variable-frequency motor; (11) pressure stabilizer; $(12,13)$ ball valve; (14) hydrophone.

due to the thermodynamic effect caused by cavitation. Besides, during the testing of interior cavitation noise, the pressure stabilizer can play the role of sound insulation and noise elimination with improved acoustic impedance. Also, it can solve the resonance and frequency-interference problems between the noise generated by the change of flowmeter and valve opening in the downstream of pressure stabilizer and the cavitation-induced noise of the pump. To reduce the interference from the system vibration, the tested pump and the motor are fixed on the huge base, and the thick rubber gaskets are used at the connections between the pipeline and the pump.

Before the testing, the cavitation tank was filled with water through the injection hole with all valves open in the closed loop system. And they were closed when there was water flowing out from the exhaust port. Then, with the inlet valve kept fully open, the air valve was open to connect the cavitation tank to the atmosphere. And, the system was stabilized after discharging the air in the system driven by variable-frequency motor. Under the rotating speed, the pump was stably operated at some flow conditions by adjusting the outlet valve in the downstream of pressure stabilizer. Further at some flow condition, the vacuum pump was turned on to reduce the pressure in the cavitation tank, and then the cavitation performance and liquid-borne noise measurements were synchronously performed.

For the liquid-borne noise measurement, a INV3020C high-performance data acquisition system combined with a 
RHSA-10 hydrophone was used. Owing to easy influence from the pressure fluctuation of pump outlet, the hydrophone was flush-mounted at 8 times the outlet diameter from the discharge flange. Different from the spectrum distribution of mechanical failure signal, the broadband feature under cavitation reflects both in low- and highfrequency bands. To ensure the spectral precision in lowfrequency band and reflect the changing trend in highfrequency band, the vibration and noise signals were collected for $30 \mathrm{~s}$ with a sampling frequency of $10.24 \mathrm{kHz}$. The instruments used in the measuring system are given in Table 2. The installation locations of the sensors are shown in Figure 3.

3.3. Cavitation Status Determination. Johann [20] pointed out that the $\mathrm{NPSH}_{1}\left(1 \%\right.$ head drop) and $\mathrm{NPSH}_{3}(3 \%$ head drop) can be used as standards for detecting the cavitation status in pumps. Based on these, the whole cavitation process is normally divided into three stages [13]: the noncavitation stage (head drop is less than 1\%), the inception cavitation stage (head drop is between $1 \%$ and $3 \%$ ), and the severe cavitation stage (head drop is higher than 3\%). To accurately obtain the cavitation numbers corresponding to $1 \%$ and $3 \%$ drop of head, the multimeasurement method was adopted during the test. Eventually, the average cavitation performance curves of the centrifugal pump under three typical flow rates were obtained, as shown in Figure 4. The corresponding cavitation numbers for different stages are shown in Table 3.

The frequency spectrum of liquid-borne noise under different cavitation stages after Fourier transform is shown in Figure 5 at a rated flow rate of $12.5 \mathrm{~m}^{3} / \mathrm{h}$. The logarithmic form is adopted in the abscissa, which can both reflect the variation law of discrete eigenvalues in low-frequency band and the overall change trend in high-frequency band. At the noncavitation stage $(\sigma=0.048)$, the noise energy is mainly concentrated in the middle- and low-frequency bands below $1000 \mathrm{~Hz}$. Moreover, it exhibits obvious discrete characteristics of APF, BPF, and their harmonics. A small number of eigenvalues appear in the frequency band of $1000 \sim 3000 \mathrm{~Hz}$, and the energy fluctuates with the increase of frequency. Compared with the noncavitation stage, the frequency spectrum at the inception cavitation stage $(\sigma=0.021)$ shows no obvious change, whereas the wideband SPL above $1000 \mathrm{~Hz}$ increases slightly. In this stage, the pressure pulsation in two-phase flow varies somewhat due to the sound energy generated by the collapse of a small amount of cavitation bubbles. However, due to the small volume and number of the cavitation bubbles, the variation is small. The main changes are concentrated in higher frequency band, which presents broadband characteristics. At the severe cavitation stage $(\sigma=0.018)$, the SPL of cavitation noise increases obviously. When the number of cavitation increases to a certain extent, the flow passage is partially blocked. Therefore, the SPLs of BPF and its harmonics caused by rotor-stator interaction decrease slightly. The high-frequency discrete signals are gradually submerged in the wide frequency band, resulting in the reduction of spectrum width.

\section{Cavitation Status Recognition}

4.1. Wavelet Packet Decomposition in Time-Frequency Domain. From Figure 5, we can see the frequency spectrums of cavitation noise under different cavitation conditions are mainly characterized by broadband. Simply studying the amplitude at a certain characteristic frequency or the overall energy value cannot accurately reflect the characteristics of cavitation noise. Therefore, the timefrequency characteristic analysis in sub-bands is of great significance for cavitation status determination. Compared with the Fourier transform, the wavelet transform can finely observe the signal detail in time-frequency domain by decomposing the time-domain signals into independent frequency bands on the basis of multiscale orthogonal refinement. Moreover, the wavelet packet transform can further decompose the high-frequency part, which is not subdivided in the wavelet transform. Considering the APF and sampling frequency are $49 \mathrm{~Hz}$ and $10.24 \mathrm{kHz}$, respectively, the $\mathrm{db} 4$ basis function was employed to proceed 6-layer wavelet packet decomposition for interior cavitation noise signals. After that, the cavitation noise signals were decomposed into 64 frequency bands, that is, the frequency bandwidth of each node is $80 \mathrm{~Hz}$ including APF. The timefrequency of cavitation noise under different cavitation stages at the rated flow rate is shown in Figure 6.

It can be seen in the time domain the cavitation noise signal presents an obvious periodicity at APF, BPF, and their harmonics with similar amplitude for different periods under the noncavitation stage. With decreasing cavitation number, the sound pressure amplitude increases, the APF becomes more difficult to identify, and the amplitude between different periods varies greatly. That is to say, the cavitation not only leads to an increase of the sound pressure amplitude but also worsens the signal periodicity and aggravates the confusion degree. In the frequency domain, a smaller peak value appears at $1000 \mathrm{~Hz}$ under the noncavitation stage. At the inception cavitation stage, the full band energy goes up, the discrete eigenvalues at BPF and its harmonics caused by rotor-stator interaction decline, and the side lobe sound pressure of discrete eigenvalue increases. In the severe cavitation stage, the energy of the frequency band below 2APF lowers, the band energy between 2APF and $2 \mathrm{BPF}$ ascents obviously, and a large number of characteristic frequency with peak value appears. These frequencies are induced by the pressure fluctuation radiated by the collapse of bubble groups and the vibration generated on the pipe wall. At $1000 \mathrm{~Hz}$, the peak value shows broadband characteristic and the energy increases at higher frequencies with no apparent peak.

\subsection{Feature Extraction Based on Frequency-Division} Statistics. The frequency band studied directly affects the accuracy of subsequent feature extraction. The wider frequency range will lower the frequency resolution. On the contrary, more eigenvalues would appear for a narrow frequency range, which will lead to more analysis time and easy data redundancy. Obviously, the 64 frequency bands 
TABLE 2: Instruments in the measuring system.

\begin{tabular}{lccc}
\hline Device & Range & Resolution & Locations \\
\hline Electromagnetic flowmeter & $1-50 \mathrm{~m}^{3} / \mathrm{h}$ & $\pm 0.5 \%$ & The length of straight pipe before and after the \\
Pressure transducer at inlet & $-0.1-0.1 \mathrm{MPa}$ & $\pm 0.5 \%$ & 2 times of pipe diameter from inlet flange \\
Pressure transducer at outlet & $0-0.1 \mathrm{MPa}$ & $\pm 0.5 \%$ & 2 times of pipe diameter from outlet flange \\
Hydrophone & $20 \mathrm{~Hz}-100 \mathrm{kHz}$ & $\pm 2 \mathrm{~dB}$ & 8 times of pipe diameter from outlet flange \\
\hline
\end{tabular}

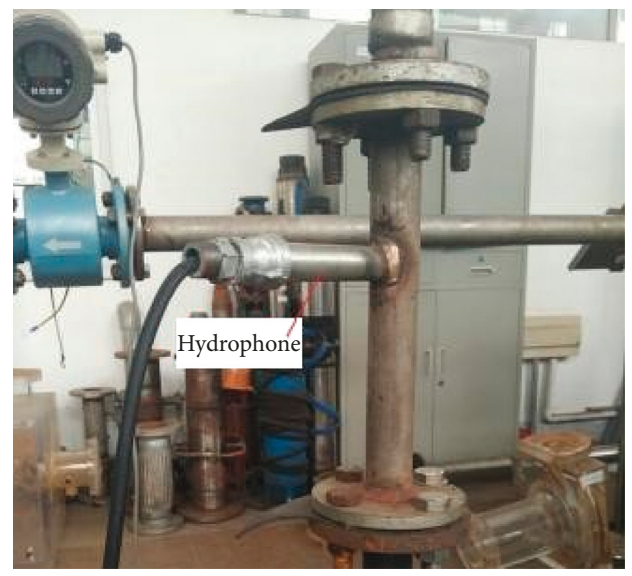

Figure 3: Positions for flow-borne noise measurement.

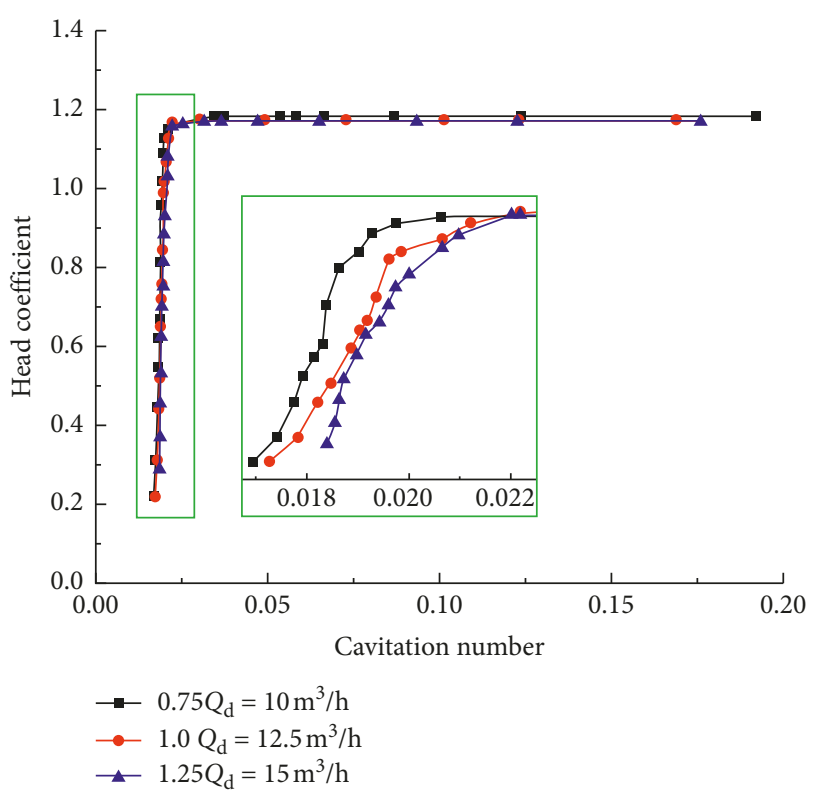

Figure 4: Cavitation performance curves under three flow rates.

decomposed by the 6-layer wavelet packet are too fine to be used as the feature frequency band. To further accurately divide the frequency bands, the energy values of each frequency band was calculated and normalized into energy coefficients. If the energy coefficient of a node is higher, it means that the node takes a larger proportion of the whole signal and contains more cavitation characteristic information. The energy coefficients under different cavitation numbers at rated flow rate are shown in Figure 7.
TABLE 3: Cavitation numbers for different cavitation stages.

\begin{tabular}{lccc}
\hline $\begin{array}{l}\text { Flow rate } \\
\left(\mathrm{m}^{3} / \mathrm{h}\right)\end{array}$ & Noncavitation & $\begin{array}{c}\text { Cavitation stage } \\
\text { Inception } \\
\text { cavitation }\end{array}$ & Severe cavitation \\
\hline 10 & $>0.020$ & $0.018-0.020$ & $<0.018$ \\
12.5 & $>0.022$ & $0.019-0.022$ & $<0.019$ \\
15 & $>0.022$ & $0.020-0.022$ & $<0.020$ \\
\hline
\end{tabular}

As can be seen, the peak energy coefficient for all frequency bands is mainly concentrated in the first 15 nodes on the sixth level, and other nodes account for a smaller amount of energy. Therefore, the discrete nodes on the 6th layer with larger energy coefficient $((6,0)-(6,7),(6,12)$, and $(6,13))$ were selected as research subject. In addition, merged with the nodes with smaller energy ratios, the upper layer nodes were chosen, eventually the nodes $(4,2),(5,7),(2,1)$, and $(1$, 1) were studied too. The optimal wavelet tree and corresponding frequency bands for each node are obtained by combining Shannon entropy, as shown in Figure 8. The time-domain feature parameters (the mean value, the root mean square, and the standard deviation) and the wavelet packet feature parameters (the energy value, the energy coefficient, and the energy entropy) extracted from the obtained cavitation noise signals at rated flow rate are used for pattern recognition in this paper. These six-feature parameters on 14 nodes (denoted as one set) constitute the inputs to the RBF neural network, and the output values represent the classification and recognition results of the cavitation status.

4.3. Feature Reduction Based on PCA. Due to large difference existing between eigenvalue elements, the $6 \times 14$ eigenmatrix obtained by the wavelet packet decomposition is not conducive for RBF neural network establishment. At the meantime, in this work, 600 sets of flow-borne noise data under different flow conditions were selected, and 84 eigenvalues were prepared for establishing the RBF network. The dimensionality reduction was carried out by the PCA method, as shown in Figure 9. After the normalized $600 \times 84$ eigenvalues were input into PCA, the eigenvectors arranging from largest to smallest were obtained, that is, $\lambda=$ (141.1170369, 76.39322079, 62.269420261, ..., 0.000204067, $0.000175016,0.00012405)$. The contribution rate of each eigenvalue $P_{\lambda}$ was calculated according to formula (7). The threshold $\theta$ is usually equal or greater than 0.9 by experience. Here, $\theta$ was selected as $97 \%$. When the sum of $k$ contribution rates is greater than $97 \%$, it is considered that it can better express the characteristics of all original data. The original 


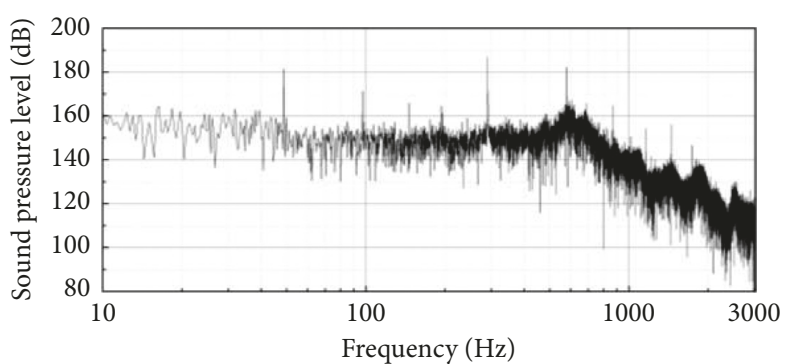

(a)

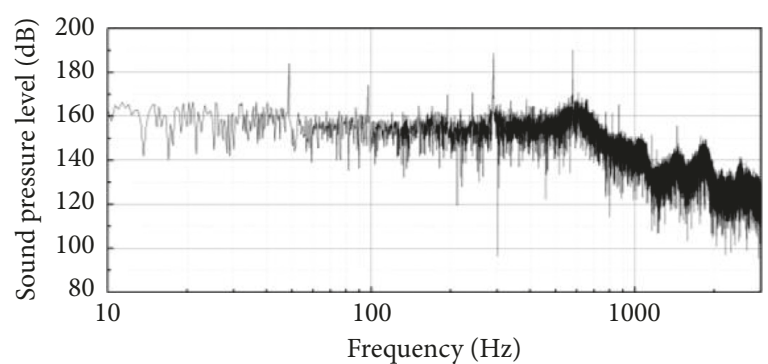

(b)

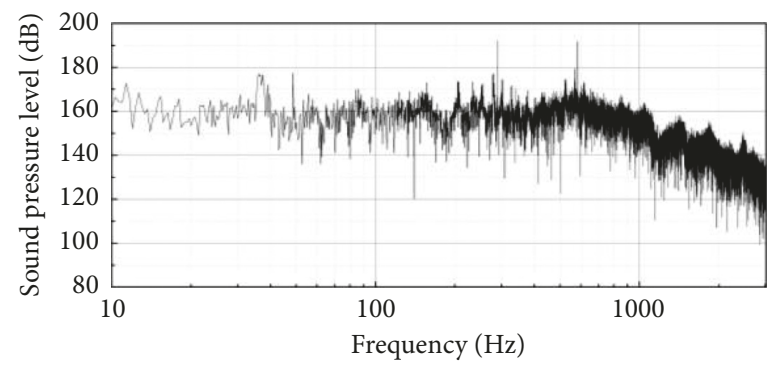

(c)

Figure 5: Frequency spectrum of cavitation noise at rated flow rate: (a) noncavitation stage $\sigma=0.048$; (b) inception cavitation stage $\sigma=0.021 ;$ (c) severe cavitation stage $\sigma=0.018$.

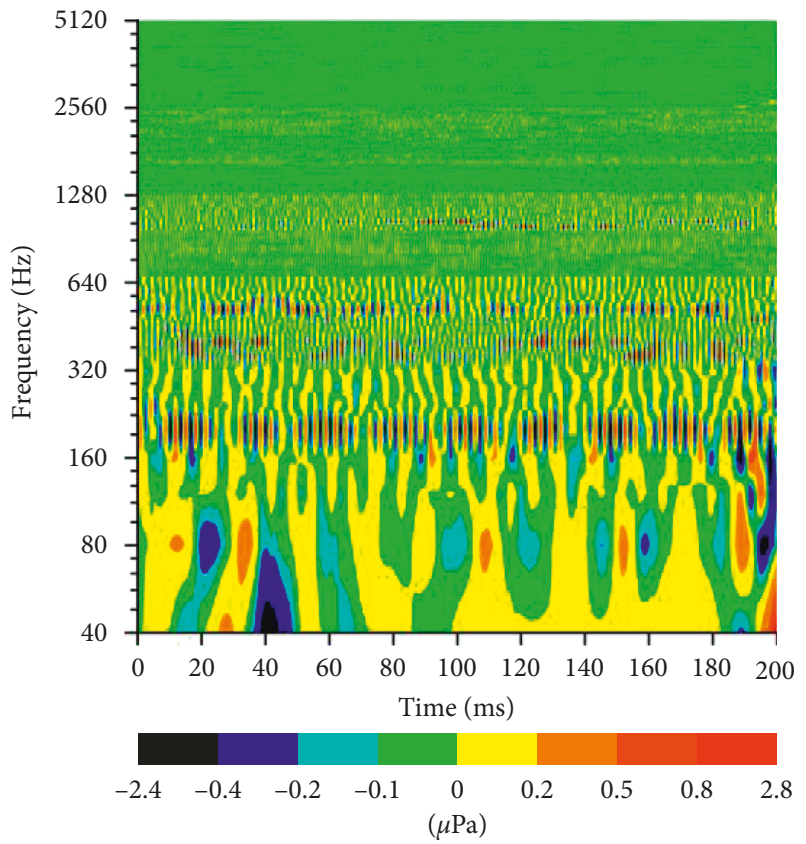

(a)

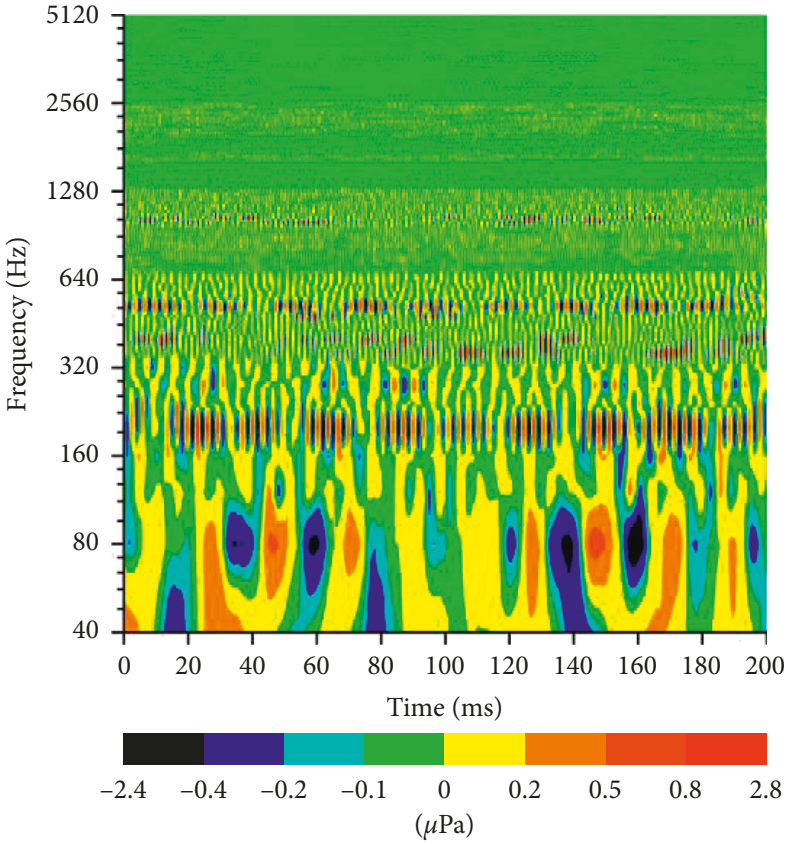

(b)

FIgURE 6: Continued. 


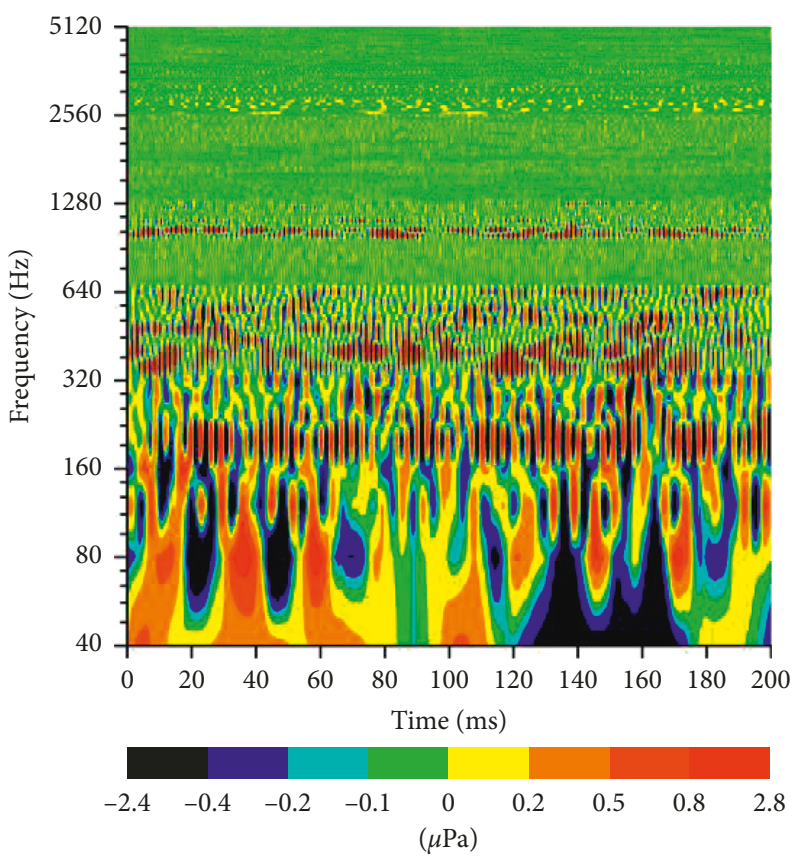

(c)

Figure 6: Time-frequency domain diagram of cavitation noise at rated flow rate: (a) noncavitation stage $\sigma=0.048$; (b) inception cavitation stage $\sigma=0.021$; (c) severe cavitation stage $\sigma=0.018$.

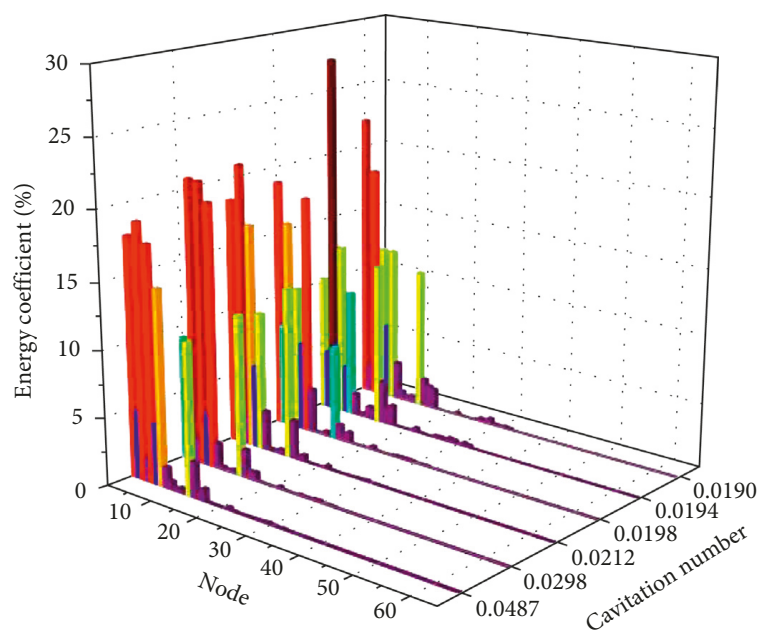

Figure 7: Energy coefficient under different cavitation numbers at rated flow rate.

$600 \times 84$ eigenvalues were then reduced to $600 \times k$ principal element eigenvalues. Here, the number of the reduced features $k$ after calculation equals to 20 .

The contribution rate of eigenvalue is calculated as follows:

$$
P_{\lambda}(i)=\frac{\lambda_{i}}{\sum \lambda_{i}} .
$$

The space distribution of principal components of fluidborne noise is shown in Figure 10. As can be seen, the bubbles under the stages of noncavitation, inception cavitation, and serious cavitation are distributed in different spatial regions with no overlap between them. It proves that the principal components obtained by PCA can be used as a good feature for different cavitation stages.

4.4. Establishment of RBF Neural Network. The RBF network typically has three layers: an input layer, a hidden layer, and an output layer. Obviously, the output nodes have been determined, which are noncavitation stage $(0,0,1)$, inception cavitation stage $(0,1,0)$, and severe cavitation $(1,0$, $0)$. In general, the number of hidden layer nodes $m$ can be firstly calculated as follows:

$$
m=\sqrt{n+1}+\alpha,
$$

where $n$ is the number of input nodes and $\alpha$ is the constant between 1 and 10 .

The approximate range calculated is from 5 to 15 . Then, after repeated attempts and verification, the suitable number of hidden layer nodes is finally determined to be 10 . Table 4 shows the input samples and the arranged outputs.

\section{Results and Discussion}

In this work, 360 sets of feature data are randomly selected as training samples, 120 sets of data are selected as verification samples, and the other 120 sets of data are used as testing samples. The identification rate will vary due to random selection of training and testing samples. In order to reduce the effect of selected samples, we run the program ten times. The average identification result is shown in Figure 11. 


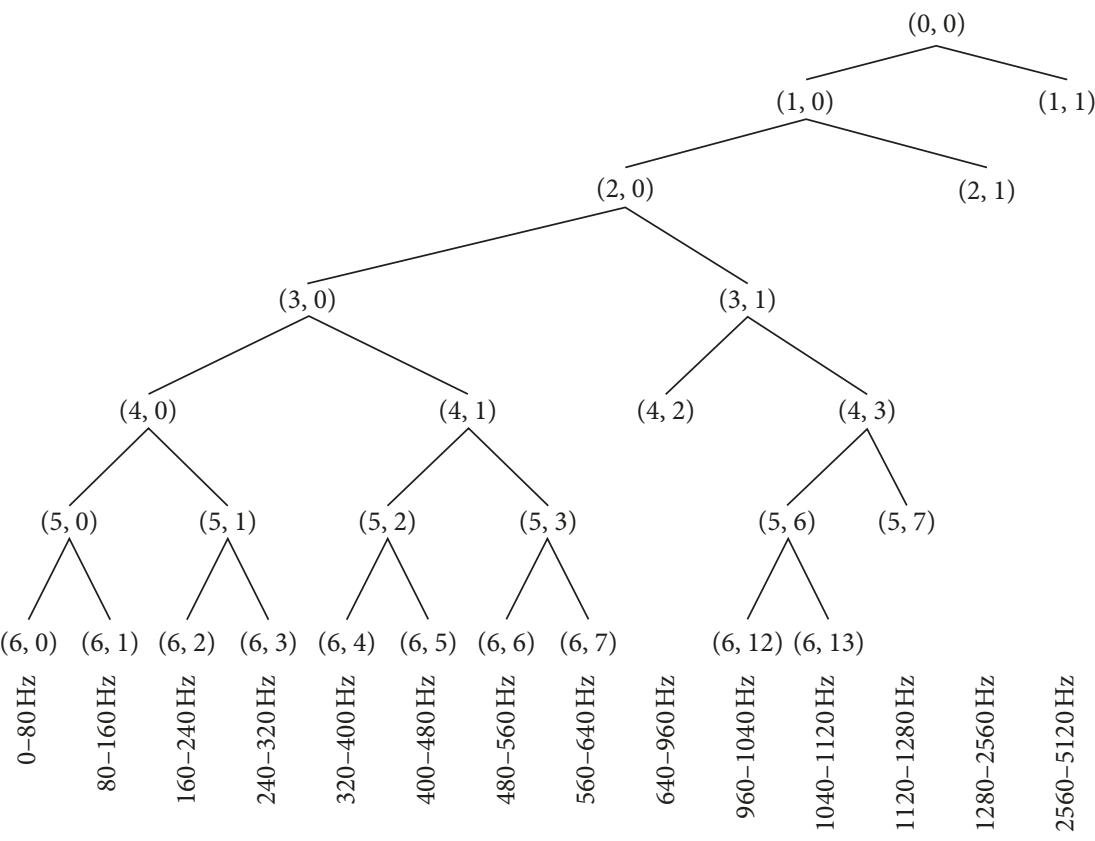

FIGURE 8: Optimal wavelet tree and corresponding frequency bands for each node.

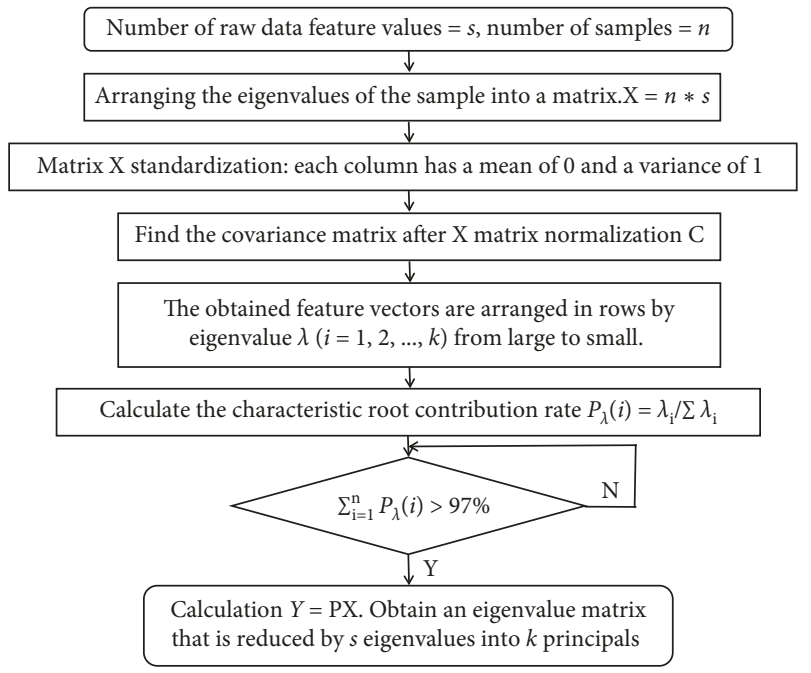

FIGURE 9: Flow chart of PCA.

As can be seen, from the perspective of the overall sample, the comprehensive identification rate for all cavitation status is higher, that is $98.2 \%$. It indicates that the method based on WPD-PCA-RBF can accurately detect the cavitation status. The identification rate of noncavitation status under different flow rates is excellent, reaching a maximum of $100 \%$. It indicates when the cavitation occurs, and it can be fully recognized by the method. The identification rate of inception cavitation status is $83.3 \%$. Four of five sets of wrong samples are misjudged as noncavitation status, and one set is misjudged as serious cavitation status. It shows the inception cavitation stage cannot be completely distinguished from the other two stages. The identification rate of serious cavitation status is $97.9 \%$. Six wrong sets of 290 sample data are misjudged as inception cavitation. It shows that the serious cavitation stage is well separated from

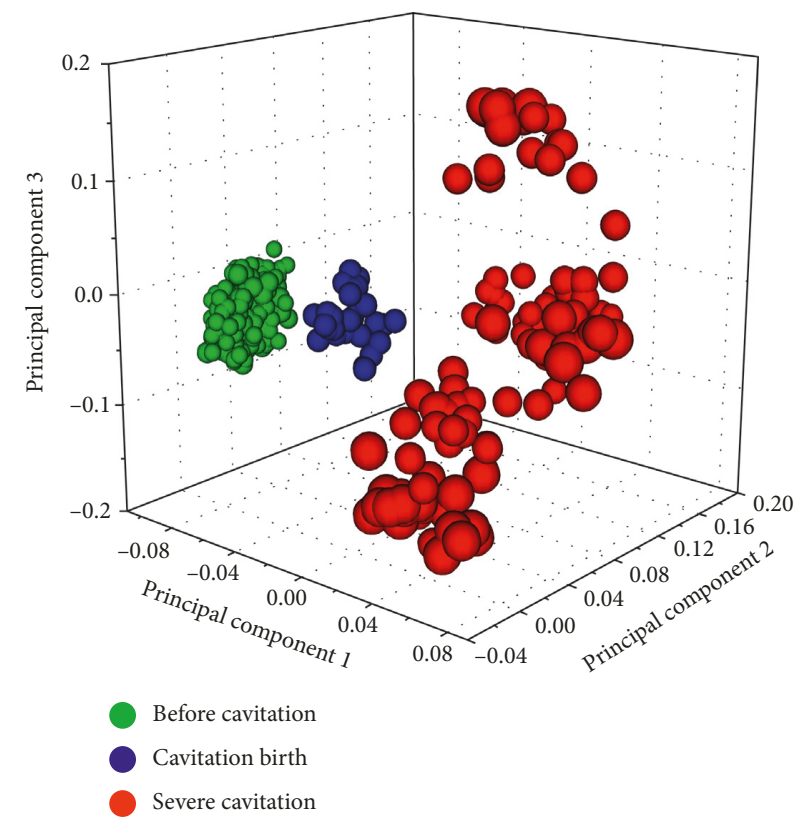

FIGURE 10: Space distribution of principal components based on PCA.

TABLE 4: Input samples and arranged outputs.

\begin{tabular}{lcccc}
\hline Flow rate $\left(\mathrm{m}^{3} / \mathrm{h}\right)$ & 10 & 12.5 & 15 & Output result \\
\hline Noncavitation & 100 & 80 & 100 & $(0,0,1)$ \\
Inception cavitation & 10 & 10 & 10 & $(0,1,0)$ \\
Serious cavitation & 90 & 110 & 90 & $(1,0,0)$ \\
\hline
\end{tabular}

the noncavitation status. The distinction from inception cavitation status needs to be improved. From the perspective of training sets, the identification rates of the three stages of noncavitation, inception cavitation, and serious cavitation 


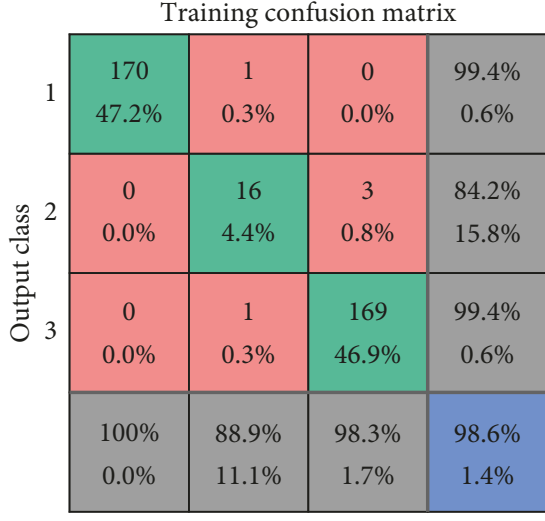

2

Target class

(a)

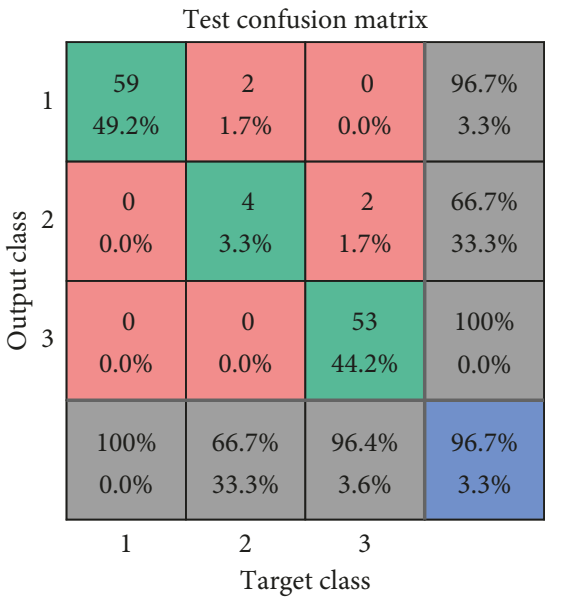

(c)

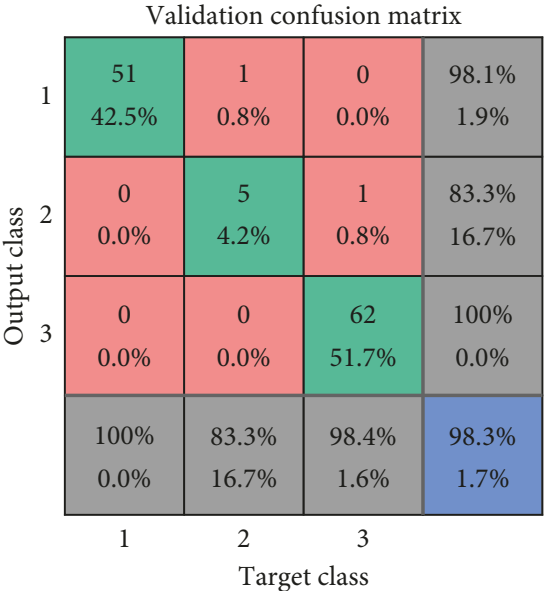

(b)

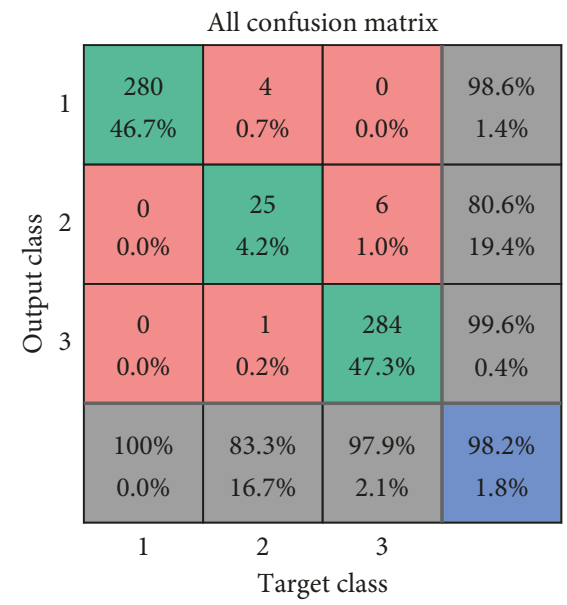

(d)

FIgURE 11: Cavitation identification result based on WPD-PCA-RBF.

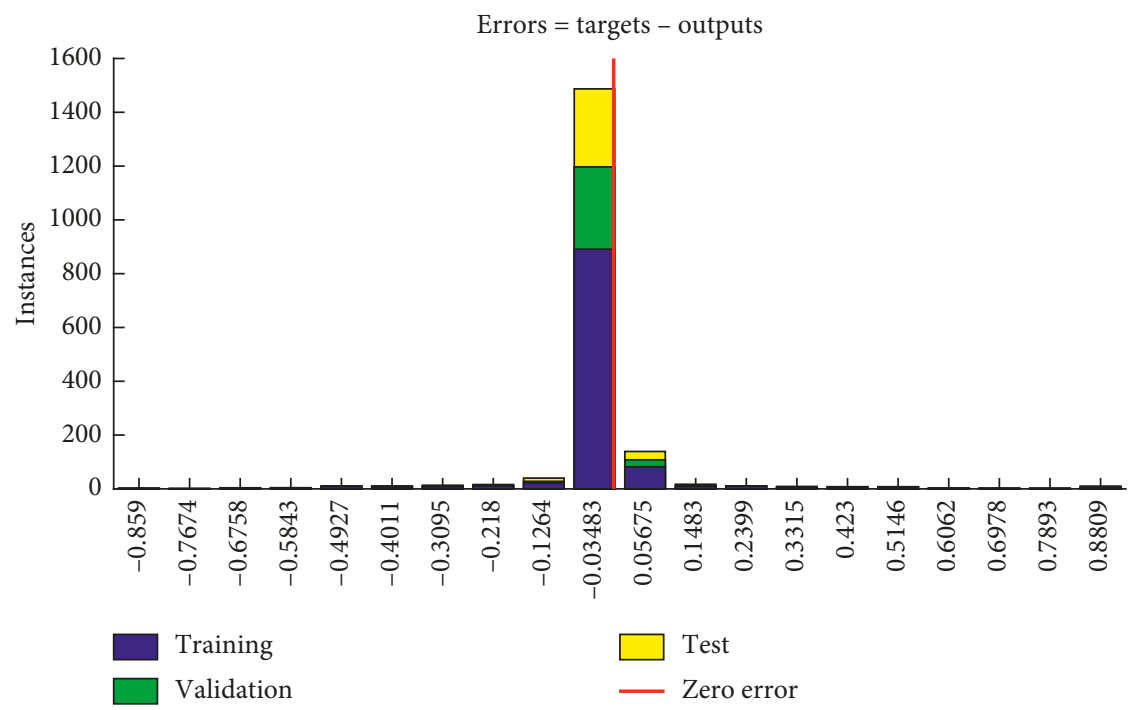

FIGURE 12: RBF neural network error distribution. 
are $100 \%, 88.9 \%$, and $98.3 \%$, respectively. It shows the eigenvalues inputted into the RBF neural network can reflect the difference of noise signals between cavitation and noncavitation statuses. However, the difference of noise signals between inception cavitation and serious cavitation statuses is not obvious.

The identification error is quantitative, and the error distribution histogram is shown in Figure 12. The red vertical line denotes zero error. The farther away from the zero-error line indicates the larger error value and less accurate identification result. It can be seen from Figure 12 that, most of the sample data are distributed in two adjacent intervals of the zero-error line $(-0.035,0.057)$. The error of a small amount of data reaches \pm 0.15 . The rest of the interval samples distribution is minimal. It shows that the identification accuracy of cavitation status is high.

\section{Conclusions}

This paper presents a cavitation status detection method suitable for centrifugal pump based on interior flow-borne noise analysis. In this method, the WPD was used to improve the frequency domain resolution. Six-feature parameters on each frequency band with higher energy coefficient were extracted. The PCA was applied to reduce dimensionality. $600 \times 84$ eigenvalues obtained after wavelet packet decomposition were reduced to $600 \times 20$ principal components. With input samples and arranged outputs, the RBF neural network was finally used to detect cavitation status. The interior flow-borne noise at three cavitation statuses under three flow conditions was measured. Moreover, 360 sets of feature data were randomly selected as training samples, 120 sets were selected as verification samples, and the other 120 sets were used as testing samples. From the perspective of overall samples, the comprehensive identification rate for cavitation status reaches $98.2 \%$. From the perspective of training sets, the identification rates of the three stages of noncavitation, inception cavitation, and serious cavitation are $100 \%, 88.9 \%$, and $98.3 \%$, respectively. The error distribution shows high identification accuracy for cavitation status. Therefore, the presented method can be applied for online monitoring and diagnosis of pump.

\section{Data Availability}

The experimental data used to support the findings of this study were supplied by the research group of Jiangsu University under license and so they still cannot be put it into the system, and the requests for access to these data should be made to the corresponding author.

\section{Conflicts of Interest}

The authors declare that they have no conflicts of interest.

\section{Acknowledgments}

This work was supported by the National Natural Science Foundation of China (Nos. 51879122 and 51779106); the Zhenjiang Key Research and Development Plan (GY2017001 and GY2018025); the Open Research Subject of Key Laboratory of Fluid and Power Machinery; Ministry of Education, Xihua University (szjj2017-094); Sichuan Provincial Key Lab of Process Equipment and Control (GK201614 and GK201816); the Advanced Talent Foundation of Jiangsu University (15JDG052); and a project funded by the Priority Academic Program Development of Jiangsu Higher Education Institutions (PAPD).

\section{References}

[1] P. Samanipour, J. Poshtan, and H. Sadeghi, "Cavitation detection in centrifugal pumps using pressure time-domain features," Turkish Journal of Electrical Engineering \& Computer Sciences, vol. 25, no. 5, pp. 4287-4298, 2017.

[2] H. Wang and P. Chen, "Intelligent diagnosis method for a centrifugal pump using features of vibration signals," Neural Computing and Applications, vol. 18, no. 4, pp. 397-405, 2009.

[3] N. R. Sakthivel, B. B. Nair, M. Elangovan, V. Sugumaran, and S. Saravanmurugan, "Comparison of dimensionality reduction techniques for the fault diagnosis of mono block centrifugal pump using vibration signals," Engineering Science and Technology, an International Journal, vol. 17, no. 1, pp. 30-38, 2014.

[4] L. Alfayez, D. Mba, and G. Dyson, "The application of acoustic emission for detecting incipient cavitation and the best efficiency point of a $60 \mathrm{~kW}$ centrifugal pump: case study," NDT \& E International, vol. 38, no. 5, pp. 354-358, 2005.

[5] S. Farokhzad and H. Ahmadi, "Acoustic based cavitation detection of centrifugal pump by neural network," Journal of Mechanical Engineering and Technology, vol. 1, no. 1, pp. 1-5, 2013.

[6] D. B. Durocher and G. R. Feldmeier, "Predictive versus preventive maintenance-future control technologies in motor diagnosis and system wellness-future control technologies in motor diagnosis and system wellness," IEEE Industry Applications Magazine, vol. 10, no. 5, pp. 12-21, 2004.

[7] A. Hernandez-Solis and F. Carlsson, "Diagnosis of submersible centrifugal pumps: a motor current and power signature approaches," EPE Journal, vol. 20, no. 1, pp. 58-64, 2010.

[8] M. Chudina, "Noise as an indicator of cavitation in a centrifugal pump," Acoustical Physics, vol. 49, no. 4, pp. 463-474, 2003.

[9] L. Dong, Y. Q. Zhao, D. Cui, and Y. Wang, "Research on cavitation acoustic characteristics of centrifugal pump based on fluid-acoustic field coupling method," Advances in $\mathrm{Me}$ chanical Engineering, vol. 10, no. 5, pp. 1-13, 2018.

[10] Y. Liu, Y. Y. He, and D. R. Chen, "Water entropy based condition test and identification of cavitation," Journal of Mechanical Strength, vol. 1, no. 31, pp. 19-23, 2009.

[11] J. Wang, L. Pan, and S. Cao, "Wavelet transforms applied to cavitation noise analysis for hydro-turbine," Journal of $\mathrm{Hy}$ droelectric Engineering, vol. 32, no. 4, pp. 215-220, 2013.

[12] H. Q. Wang and P. Chen, "Sequential condition diagnosis for centrifugal pump system using fuzzy neural network," Neural Information Processing, vol. 11, no. 3, pp. 41-50, 2007.

[13] G. He, Y. Cao, T. Ming et al., "Cavitation state recognition of centrifugal pump based on features of modified octave bands," Journal of Harbin Engineering University, vol. 38, no. 8, pp. 1263-1267, 2017.

[14] X. G. Yang, "Pattern recognition of engine abnormal sound based on wavelet packet and bispectrum," Noise and Vibration Control, vol. 38, no. 6, pp. 146-149, 2018. 
[15] L. Su, T. Shi, Z. Liu, H. Zhou, L. Du, and G. Liao, "Nondestructive diagnosis of flip chips based on vibration analysis using PCA-RBF," Mechanical Systems and Signal Processing, vol. 85, pp. 849-856, 2017.

[16] G. L. Liao, L. Du, L. Su, M. Zeng, L. Nie, and T. Shi, "Using RBF networks for detection and prediction of flip chip with missing bumps," Microelectronics Reliability, vol. 55, no. 12, pp. 2817-2825, 2015.

[17] S. Chang, M. Leng, H. Wu, and J. Thompson, "Aircraft ice accretion prediction using neural network and wavelet packet transform," Aircraft Engineering and Aerospace Technology, vol. 88, no. 1, pp. 128-136, 2016.

[18] M. A. Halali, V. Azari, M. Arabloo, A. H. Mohammadi, and A. Bahadori, "Application of a radial basis function neural network to estimate pressure gradient in water-oil pipelines," Journal of the Taiwan Institute of Chemical Engineers, vol. 58, pp. 189-202, 2016.

[19] L. Dong, Y. Q. Zhao, and C. Dai, "Detection of inception cavitation in centrifugal pump by fluid-borne noise diagnostic," Shock and Vibration, vol. 2019, Article ID 9641478 , 15 pages, 2019.

[20] F. G. Johann, Centrifugal Pump, Springer, Berlin, Heidelberg, Germany, 2014 


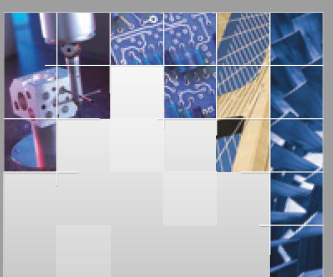

\section{Enfincering}
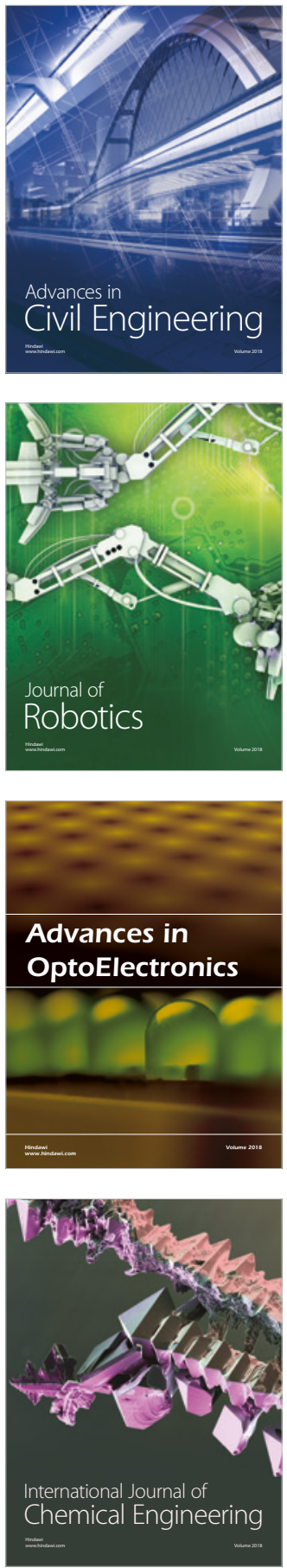

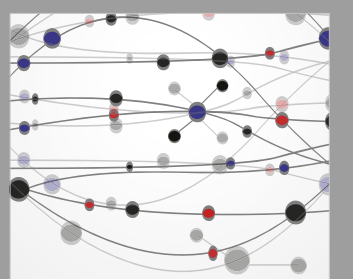

\section{Rotating \\ Machinery}

The Scientific World Journal

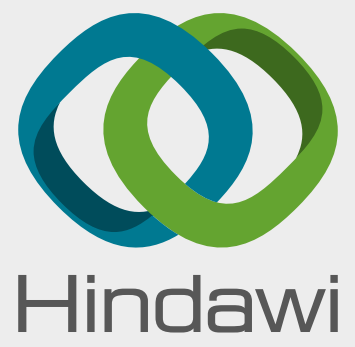

Submit your manuscripts at

www.hindawi.com
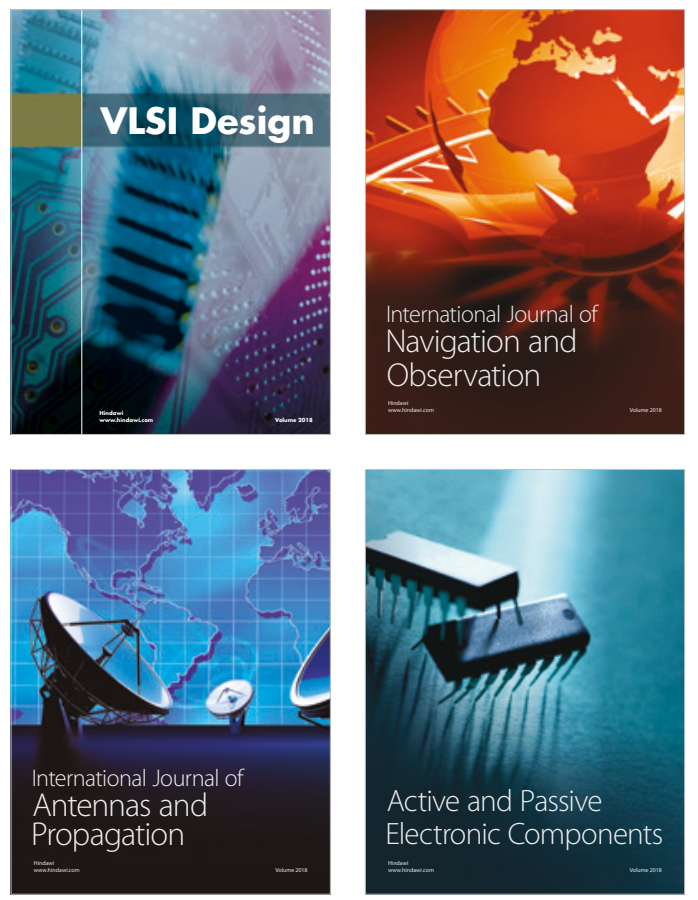
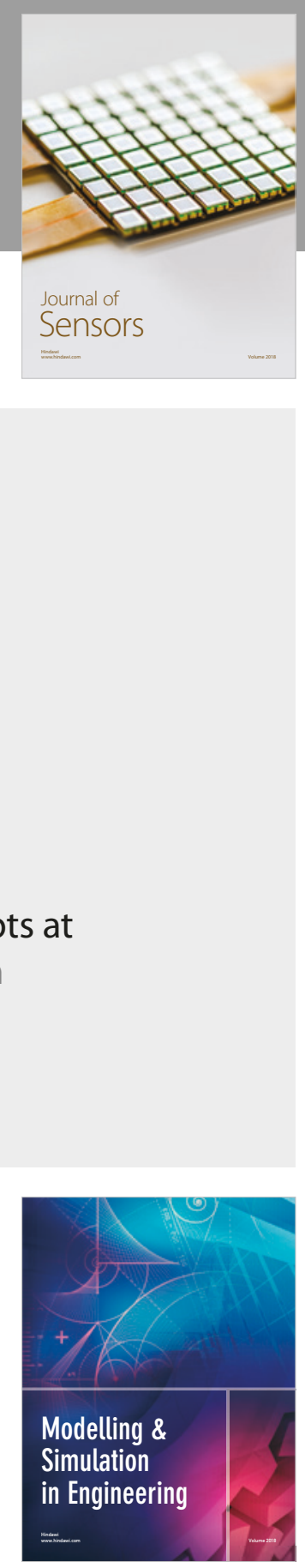

\section{Advances \\ Multimedia}
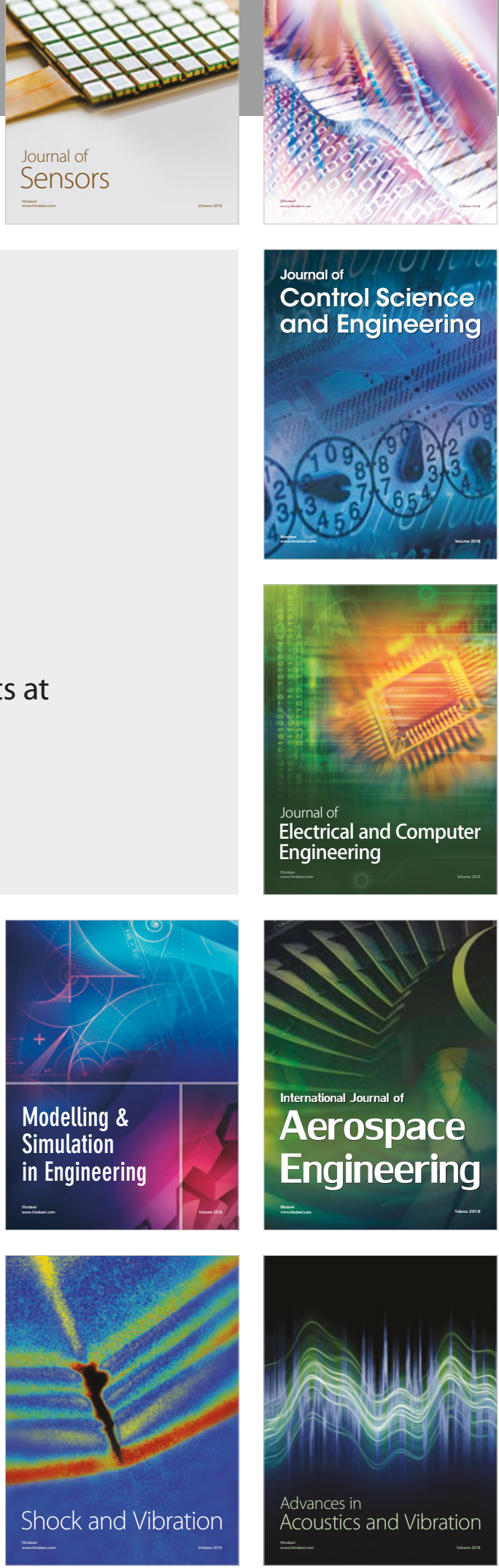